\title{
Prediction of the aggressiveness of non- functional pancreatic neuroendocrine tumors based on the dual-tracer PET/CT
}

Susanna Majala ${ }^{1,2}$ (D) Hanna Seppänen ${ }^{3}$, Jukka Kemppainen ${ }^{2,4}$, Jari Sundström5 ${ }^{5}$ Camilla Schalin-Jäntti ${ }^{6}$, Risto Gullichsen ${ }^{1}$, Jukka Schildt ${ }^{7}$, Harri Mustonen ${ }^{8}$, Tiina Vesterinen ${ }^{9,10}$, Johanna Arola ${ }^{9}$ and Saila Kauhanen ${ }^{1,2^{*}}$

\begin{abstract}
Background: Predicting the aggressive behavior of non-functional pancreatic neuroendocrine tumors (NF-PNET) remains controversial. We wanted to explore, in a prospective setting, whether the diagnostic accuracy can be improved by dual-tracer functional imaging ${ }^{68} \mathrm{Ga}$-DOTANOC and ${ }^{18} \mathrm{~F}-\mathrm{FDG}-\mathrm{PET} / \mathrm{CT}$ in patients with NF-PNETs.

Methods: Thirty-one patients with NF-PNET (90\% asymptomatic) underwent PET-imaging with ${ }^{18} \mathrm{~F}$-FDG and ${ }^{68} \mathrm{Ga}-$ DOTANOC, followed by surgery $(n=20)$, an endoscopic ultrasonography and fine-needle biopsy $(n=2)$ or followup $(n=9)$. A focal activity on PET/CT greater than the background that could not be identified as physiological activity was considered to indicate tumor tissue. The imaging results were compared to histopathology. The mean follow-up time was 31.3 months.

Results: Thirty-one patients presented a total of 53 lesions (40 histologically confirmed) on PET/CT. Thirty patients had a ${ }^{68} \mathrm{Ga}$-DOTANOC-positive tumor (sensitivity 97\%) and 10 patients had an ${ }^{18} \mathrm{~F}$-FDG-positive tumor. In addition, one ${ }^{68} \mathrm{Ga}$-DOTANOC-negative patient was ${ }^{18} \mathrm{~F}$-FDG-positive. ${ }^{18} \mathrm{~F}$-FDG-PET/CT was positive in 19\% (3/16) of the G1 tumors, $63 \%$ (5/8) of the G2 tumors and 1/1 of the well-differentiated G3 tumor. ${ }^{68} \mathrm{Ga}$-DOTANOC-PET/CT was positive in $94 \%$ of the G1 tumors, 100\% of the G2 tumors and 1/1 of the well-differentiated G3 tumor. Two out of six (33\%) of the patients with lymph node metastases (LN+) were ${ }^{18} \mathrm{~F}$-FDG-positive. The ${ }^{18} \mathrm{~F}$-FDG-PET/CT correlated with tumor Ki-67 $(P=0.021)$. Further, the Krenning score correlated with tumor Ki-67 $(P=0.013) .{ }^{18} \mathrm{~F}$-FDG-positive tumors were significantly larger than the ${ }^{18} \mathrm{~F}$-FDG-negative tumors $(P=0.012) .{ }^{18} \mathrm{~F}-\mathrm{FDG}$-PET/CT showed a positive predictive value of $78 \%$ in the detection of potentially aggressive tumors (G2, G3, or LN + PNETs); the negative predictive value was $69 \%$.
\end{abstract}

Conclusions: ${ }^{18} \mathrm{~F}-\mathrm{FDG}-\mathrm{PET} / \mathrm{CT}$ is useful to predict tumor grade but not the LN+ of NF-PNETs. Patients with ${ }^{18} \mathrm{~F}-\mathrm{FDG}$ avid NF-PNETs should be referred for surgery. The ${ }^{68} \mathrm{Ga}$-DOTANOC-PET/CT also has prognostic value since the Krenning score predicts the histopathological tumor grade.

Trial registration: The study has been registered at ClinicalTrials.gov; Non-functional Pancreatic NET and PET imaging, NCT02621541.

Keywords: NF-PNET, ${ }^{18}$ F-FDG-PET/CT, ${ }^{68}$ Ga-DOTANOC-PET/CT, prospective study, surgical management, Ki-67

\footnotetext{
* Correspondence: saila.kauhanen@utu.fi

'Division of Digestive Surgery and Urology, Turku University Hospital, P.O.

Box 52, FIN-20521 Turku, Finland

${ }^{2}$ Turku PET Centre, Turku University Hospital, P.O. Box 52, FIN-20521 Turku,

Finland

Full list of author information is available at the end of the article
} 


\section{Background}

Pancreatic neuroendocrine tumors (PNETs) constitute 3\% of all pancreatic neoplasms and $60-80 \%$ of PNETs are defined as non-functional (NF-PNET) [1]. The incidence has increased in recent years due to the expanding use of imaging [2]. Despite the generally indolent nature, it has been recognized that the pathological potential of PNETs is highly variable and some NF-PNETs present at an advanced stage with local invasion and distant metastases [3, 4]. Heterogeneity of PNETs makes therapeutic decisions difficult with no clear consensus.

The most powerful prognostic factors are the grade and distant metastases [4]. Although complete surgical resection is the only potentially curative treatment, a conservative approach seems to be safe for asymptomatic and stable sporadic NF-PNET $\leq 2 \mathrm{~cm}[5,6]$. However, a locally advanced disease with lymph node metastases $(\mathrm{LN}+)$ is rare but also possible on small $(1-2 \mathrm{~cm})$ NF-PNETs [7]. In a review of 136 surgical patients, Hashim and colleagues suggested a metastatic rate of $8 \%$ in PNETs as small as 1.5 cm [8]. In a retrospective analysis $(n=181)$, Partelli et al. [9] demonstrated that lymph node metastases decreased the 5-year disease-free survival in NF-PNETs (70\% vs 97\%, $P<0.001)$. Pancreatic resection is a high-risk operation with $20-40 \%$ morbidity and $1-2 \%$ mortality $[10,11]$. Knowing the risks of pancreatic surgery, it is challenging to decide between surgery and follow-up for these patients.

The vast majority of well-differentiated PNETs express somatostatin receptors (SSTRs) and can be visualized by the binding of a radioactive somatostatin analog. SSTRbased functional imaging, ${ }^{68} \mathrm{Ga}$-DOTANOC positron emission tomography/computed tomography (PET/CT) has a high diagnostic sensitivity (88-100\%) for localizing NETs [12-14] but provides limited information about the aggressiveness of PNET. ${ }^{18}$ F-fluoro-2-deoxyglucose (FDG)-PET/CT demonstrates increased cellular tissue metabolism. Although the sensitivity of ${ }^{18}$ F-FDG-PET/ CT imaging is low for NETs, positive ${ }^{18} \mathrm{~F}-\mathrm{FDG}$-PET/CT predicts poor survival for NET patients [15, 16]. Dualtracer functional imaging of NF-PNET has only been studied in retrospective series $[17,18]$.

The aim of this study was to determine the role of dualtracer functional imaging in predicting aggressive behavior in NF-PNETs in a prospective setting with histopathological references. The hypothesis was that the higher the maximum standardized uptake value $\left(\mathrm{SUV}_{\max }\right)$ of ${ }^{18} \mathrm{~F}$ FDG-PET/CT then the higher the Ki-67 of the tumor would be and the higher the $\mathrm{SUV}_{\max }$ of ${ }^{68} \mathrm{Ga}$-DOTANOC-PET/CT the lower the Ki-67 of the tumor would be.

\section{Materials and methods}

\section{Study design}

The study was a prospective, multicenter clinical trial at Turku and Helsinki University Hospitals in Finland.
From January 2016 to January 2018, a total of 35 patients suspected of having NF-PNET on a primary CT were prospectively imaged using ${ }^{68} \mathrm{Ga}$-DOTANOC-PET/CT and ${ }^{18}$ F-FDG-PET/CT. Four patients were excluded; one patient diagnosed with a pancreatic adenocarcinoma, another with a functional duodenal neuroendocrine tumor (gastrinoma), one with mixed neuroendocrine non-neuroendocrine neoplasm and one patient with an uncertain diagnosis lacking pathology. A total of 31 patients (age range 20-83 years; mean age $60 \pm 18$ years) were enrolled in the study. Patient characteristics are given in Table 1 . Twenty patients were operated. Four patients underwent endoscopic ultrasonography and fine-needle biopsy (EUS-FNB), but two of these were found to be non-specific. One patient with Von Hippel-Lindau (VHL) syndrome had a pancreatic intraepithelial neoplasia lesion in the histopathological analysis and two NF-PNETs ( $\varnothing 13$ and $10 \mathrm{~mm}$ on MRI) were followed up. Twenty-two patients had a histopathological confirmation (20 resection specimens and two EUS-FNB) and nine patients with a positive ${ }^{68} \mathrm{Ga}$-DOTANOC PET/ CT finding were followed up (33.5 \pm 6.2 months). Thirtyone patients had a total of 53 lesions on the PET/CT imaging. The median PET/CT imaging interval was 34 days (range 2-164, IQR 12-62 days) in 29 patients. The PET/ CT imaging interval was delayed in two patients, in 1 to 360 days due to logistical reasons and in another to 244 days due to a more urgent operation. Since there were two methods to measure chromogranin $\mathrm{A}(\mathrm{CgA})$ in use at the laboratories during the study period, the CgA was reported as three subgroups. For an accurate diagnosis of PNETs every attempt was made to establish a tissue sample by means of an operation or an EUS-FNB. Follow-up time was measured from the date of the first PET/CT scan to the review time. Patients were treated in accordance with the routine procedures of the departments and the European Guidelines [19]. The study has been registered at Clinical Trials.gov (NCT02621541).

\section{${ }^{68} \mathrm{Ga}-\mathrm{DOT} A N O C$ and ${ }^{18} \mathrm{~F}-\mathrm{FDG}$-PET/CT protocol}

PET/CT was performed using the Discovery STE $\left(6{ }^{68} \mathrm{Ga}\right.$ DOTANOC and $14{ }^{18} \mathrm{~F}$-FDG-PET/CT $)$ or VCT $\left(11{ }^{68} \mathrm{Ga}\right.$ DOTANOC and $10{ }^{18}$ F-FDG-PET/CT) scanner (General Electric Medical Systems, Milwaukee, WI) and one patient underwent ${ }^{18}$ F-FDG-PET/(magnetic resonance imaging) MRI using an Ingenuity TF PET/MRI scanner (Phillips Medical Systems, Cleveland, $\mathrm{OH}$ ) at Turku PET center and Siemens Biograph mCT 64 (Siemens Healthineers, Erlangen, Germany) (12 ${ }^{68} \mathrm{Ga}-\mathrm{DOTANOC}$ and $4{ }^{18} \mathrm{~F}$ FDG-PET/CT) or Gemini PET-CT scanner (Philips Inc, USA) (1 ${ }^{68}$ Ga-DOTANOC and $1{ }^{18}$ F-FDG-PET/CT $)$ at Nuclear Medicine Department in Helsinki University Hospital. One ${ }^{68} \mathrm{Ga}$-DOTANOC and $1{ }^{18} \mathrm{~F}$ - FDG-PET/CT scan were done at a private institute using the Siemens Biograph 6 (Siemens Medical Solutions, USA). 
Table 1 Patient characteristics

\begin{tabular}{|c|c|}
\hline No. patients & 31 \\
\hline Gender, Male, $n(\%)$ & $20(65)$ \\
\hline Age years, mean (SD) & $59.6(17.6)$ \\
\hline BMl, mean (SD) & $26.3(4.0)$ \\
\hline Asymptomatic, $n(\%)$ & $29(90)$ \\
\hline MEN1 syndrome, $n(\%)$ & $7(23)$ \\
\hline \multicolumn{2}{|l|}{$\mathrm{CgA}, n(\%)$} \\
\hline Strongly positive & $3(10)$ \\
\hline Weakly positive & $15(48)$ \\
\hline Negative & $13(42)$ \\
\hline $\mathrm{PP}(\mathrm{pmol} / \mathrm{L})$, median (IQR) & $68(41-129)$ \\
\hline 5-HIAA (nmol/L), median (IQR) & $64(53-64)$ \\
\hline \multicolumn{2}{|l|}{ Primary tumor size (mm) } \\
\hline All patients, median (IQR) & $24(14-35)$ \\
\hline Operated, median (IQR) $(n=20)$ & $31(20-52)$ \\
\hline Biopsy and follow-up, median (min-max) $(n=2)$ & $24(22-25)$ \\
\hline Follow-up*, median (IQR) $(n=9)$ & $14(13-21)$ \\
\hline \multicolumn{2}{|l|}{ Tumor localization, $n(\%)$} \\
\hline Head & $12(39)$ \\
\hline Body & $2(6)$ \\
\hline Tail & $10(32)$ \\
\hline Multiple & $7(23)$ \\
\hline \multicolumn{2}{|l|}{ Treatment, $n(\%)$} \\
\hline Surgery & $20(65)$ \\
\hline Biopsy and follow-up & $2(6)$ \\
\hline Follow-up* & $9(29)$ \\
\hline \multicolumn{2}{|l|}{ Type of surgery, $n(\%)$} \\
\hline Total pancreatectomy & $2(10)$ \\
\hline Pancreaticoduodenectomy & $4(20)$ \\
\hline Distal pancreatectomy & $13(65)$ \\
\hline Enucleation & $1(5)$ \\
\hline \multicolumn{2}{|l|}{ Type of surgery, n (\%) } \\
\hline Open & $13(75)$ \\
\hline Laparoscopic & $5(25)$ \\
\hline Robotic surgery & $2(10)$ \\
\hline \multicolumn{2}{|l|}{ Grade, $n(\%)$} \\
\hline G1 & $13(59)$ \\
\hline G2 & $8(36)$ \\
\hline G3 NET & $1(5)$ \\
\hline G3 NEC & 0 \\
\hline
\end{tabular}

Abbreviations: $\mathrm{CgA}$, circulating chromogranin $\mathrm{A}$, strongly positive indicates $\mathrm{S}$ $\mathrm{CgA}=13.5 \mathrm{nmol} / \mathrm{L}$ or $\mathrm{P}-\mathrm{CgA} 9-37 \mathrm{nmol} / \mathrm{L}$, weakly positive indicates $\mathrm{S}-\mathrm{CgA} 2.2-$ $4.7 \mathrm{nmol} / \mathrm{L}$ or $\mathrm{P}-\mathrm{CgA} 3.0-4.8 \mathrm{nmol} / \mathrm{L}$ and negative indicates $\mathrm{S}-\mathrm{CgA}<2.1 \mathrm{nmol} / \mathrm{L}$ or P-CgA $<3.0 \mathrm{nmol} / \mathrm{L} ; B M I$, body mass index, $\mathrm{kg} / \mathrm{m}^{2} ; M E N 1$, multiple endocrine neoplasia type 1 syndrome; $P P$, pancreatic polypeptide; 5-HIAA, 5 -hydroxyindoleatic acid

*Two patients underwent non-diagnostic EUS-FNB (endoscopic ultrasonography and fine needle biopsy)
Patients underwent a whole-body PET/CT scan from the level of the skull base to the mid-thigh starting $64 \pm 13 \mathrm{~min}$ after the injection of ${ }^{68} \mathrm{Ga}$-DOTANOC and $55 \pm 9$ min after ${ }^{18} \mathrm{~F}$-FDG. The mean dose of intravenous ${ }^{68} \mathrm{Ga}$-DOTANOC was $143.8 \pm 17.1 \mathrm{MBq}$ and ${ }^{18} \mathrm{~F}$-FDG was $321.9 \pm 67.3 \mathrm{MBq}$. The patients fasted for $6 \mathrm{~h}$ before the study. Blood glucose levels were checked before any ${ }^{18}$ F-FDG-PET/CT for patients with diabetes or previous history of glucocorticoids use (range 4.6-8.3 mmol/l). A low-dose PET/CT was followed with a whole-body diagnostic CT scan after automated intravenous injection of the contrast agent, either with ${ }^{68} \mathrm{Ga}$-DOTANOC- or ${ }^{18} \mathrm{~F}$-FDG-PET/CT. Attenuation correction was performed using a low-dose ultrafast CT protocol ( $80 \mathrm{mAs}, 140 \mathrm{kV}, 0.3 \mathrm{mSv}$ per field of view). Images were reconstructed full width at half maximum and fully three-dimensional maximum-likelihood ordered-subset expectation maximization (OSEM). Data was corrected for dead time, decay, and photon attenuation and was reconstructed to a $128 \times 128$ matrix.

\section{Data analysis}

The diagnostic accuracy of the PET/CT studies was assessed by comparing the PET-images and the histopathological reports $(n=22)$. When proper histology was not available $(n=9)$, the consensus was based on the sum of the laboratory tests and imaging procedures. This information was used for the interpretation of the lesion analysis (Figs. 1 and 2). Data on the primary tumor site and the diameter were collected from the pathology reports and from other imaging studies (contrast CT or MRI). The histopathological analysis was blind and conducted by two experienced pathologist (J.A and J.Su). Clinical TNM and grade classification was based on the 2017 World Health Organization (WHO) classification of PNETs [5].

Only the patients and the tumors with a histopathological confirmation were included in the correlation analysis. Ki-67-labeling indexes were studied from the whole series of tumors using MIB-I antibody (Dako, Agilent Pathology Solutions, Santa Clara, CA, USA) and automated staining instrument (BenchMark Ultra, Ventana Medical Systems, Inc., Tucson, AZ, USA). All stainings were prepared in the clinical pathology laboratory (HUSLAB, Helsinki University Hospital) under standardized conditions. Stained slides were digitized with a Panoramic scanner (3DHISTECH, Budapest, Hungary) and $\mathrm{Ki}-67$ values were calculated by T.V from hot spot areas comprising of at least 2000 cells. A publicly available application that was shown suitable for pancreatic NETs, was used for quantitative image analysis (ImmunoRatio) [20, 21].

Analyses of PET/CT-images were interpreted by a dedicated nuclear medicine physician (J.K), with short referral information but blinded to histopathological 


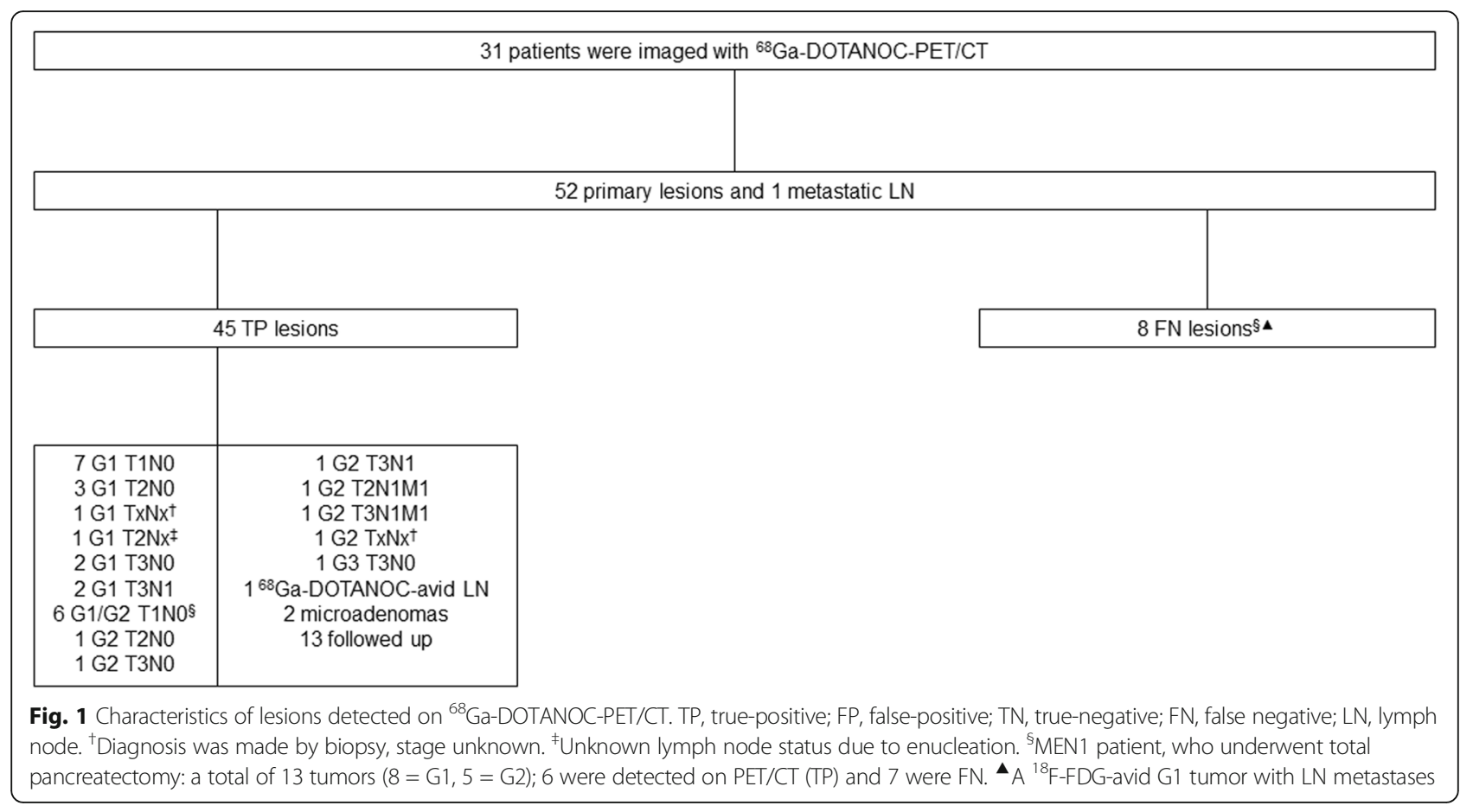

report. The $\mathrm{SUV}_{\max }$-values were determined for every tumor or abnormal anatomical region on both ${ }^{68} \mathrm{Ga}$ DOTANOC and ${ }^{18} \mathrm{~F}$-FDG-PET/CT. For the PET/CT-studies areas with a focal activity greater than the background that could not be identified as physiological activity were considered to indicate tumor tissue. Lesions were graded on ${ }^{68} \mathrm{Ga}$-DOTANOC-PET/CT with the Krenning score, by semi-quantitatively comparing $\mathrm{SUV}_{\max }$ of the tumors to the reference organs such as liver and spleen. Krenning scoring was performed as follows: score 1: uptake < normal liver, 2: uptake $=$ normal liver, 3: uptake $>$ normal liver and 4 : uptake $>$ spleen or kidneys [22]. Further, by using the dualtracer PET/CT the NETPET-score (grades P1-5) was defined. Grade P1 indicated purely somatostatin analog avid lesion without ${ }^{18} \mathrm{~F}$-FDG-uptake and P5 indicated the presence of a significant ${ }^{18} \mathrm{~F}$-FDG-positive and somatostatin




analog-negative diseas e[23]. The imaging analysis was performed using ADW 4.4 workstation.

\section{Statistical analysis}

Normally distributed variables were expressed as means and standard deviations (SD) variables not following a normal distribution as medians and interquartile ranges (IQR) and categorical variables as frequencies and proportions. The Shapiro-Wilk test was used to test deviations from a normal distribution. Spearman's rank correlation was used to test the relationship between Ki-67 and $\mathrm{SUV}_{\max }$ due to a lack of normally distributed data. The Mann-Whitney or the Kruskal-Wallis tests were used to discover the differences between the groups in continuous variables; Fisher's exact test was used for binary variables and the linear by linear association test for ordinal variables. A $P$ value of $<$ 0.05 was considered statistically significant, and two-tailed tests were used. The data analysis was performed using commercially available software (Statistical Package for Social Sciences, version 24, IBM Corp., Armonk, NY, USA).

\section{Results}

Features of NF-PNETs and histopathological grading

The type of surgery and histopathological finding of the tumors are presented in Table 1. Patients with G1 NFPNET were more commonly asymptomatic (92\%) compared to $\mathrm{G} 2$ patients (75\% asymptomatic), but the only patient with a G3 tumor was also asymptomatic $(P=$ 0.595). One symptomatic patient had jaundice and two had upper abdominal pains. All the symptomatic patients had sporadic NF-PNET.

Multiple endocrine neoplasia 1 (MEN1) patients $(n=7)$ more commonly had a G1 (71\%) rather than a G2 (29\%) tumor and none had a G3 tumor, but the difference was not statistically significant $(P=0.470)$. There was no correlation between the tumor size and the grade: G1 (34 \pm $27 \mathrm{~mm}), \mathrm{G} 2(36 \pm 28 \mathrm{~mm})$, and G3 $(n=1,45 \mathrm{~mm})(P=$ $0.800)$. However, all the operated primary tumors $\leq 2 \mathrm{~cm}$ $(n=6)$ were G1 tumors without lymph node metastases. The most common location for the G1 tumor was the tail of the pancreas (54\%). Three out of eight (38\%) of the G2 NF-PNETs were located in the head and the same amount; $3 / 8$ (38\%) in the tail of the pancreas. The G3 NFPNET was located in the head of the pancreas. There was no statistical significance between the location of a tumor and the grade of a tumor $(P=0.554)$. There was a trend between the grade and CgA $(P=0.108)$.

\section{Correlation between ${ }^{68} \mathrm{Ga}$-DOTANOC-PET/CT and grade}

${ }^{68} \mathrm{Ga}$-DOTANOC-PET/CT was positive in 30 patients and thus the sensitivity of the ${ }^{68} \mathrm{Ga}$-DOTANOC-PET/CT detecting of NF-PNETs was $97 \%$ and specificity was $100 \%$. The only ${ }^{68} \mathrm{Ga}$-DOTANOC-negative tumor (G1) was ${ }^{18} \mathrm{~F}$ FDG-positive and the patient had multiple (11/13) lymph node metastases. Thirty-one study patients had a total of 53 lesions, 45 of these were ${ }^{68} \mathrm{Ga}$-DOTANOC-positive (32/45 histologically confirmed lesions). Eight lesions were not detected by PET/CT at all and were false negative (FN) lesions (Fig. 1); one patient had negative ${ }^{68} \mathrm{Ga}$ DOTANOC-PET/CT and histologically confirmed G1 NET and seven FN lesions were detected on the same MEN1 patient who underwent a total pancreatectomy. $\mathrm{He}$ had a total of 13 PNETs (five G2 T1N0 tumors and eight G1 T1N0 tumors). Six of these lesions were detected on ${ }^{68} \mathrm{Ga}$-DOTANOC-PET/CT and one of these tumors was also ${ }^{18} \mathrm{~F}$-FDG-positive. Three lesions were $>10 \mathrm{~mm}$ and 10 were $5-10 \mathrm{~mm}$ in size. There were also multiple microadenomas $(\mathrm{NET}<5 \mathrm{~mm})$. Due to several lesions, it was impossible to combine the information concerning the 13 tumors on a histopathological report and the six lesions on ${ }^{68} \mathrm{Ga}$-DOTANOC-PET/CT. Only the largest primary tumor was included in the lesion analysis.

The lesion-based sensitivity of ${ }^{68} \mathrm{Ga}$-DOTANOC-PET/ CT to detect of NF-PNETs was $85 \%$ with a specificity of $100 \%$. There was no correlation between the $\mathrm{SUV}_{\max }$ of ${ }^{68} \mathrm{Ga}$-DOTANOC-PET/CT and the tumor Ki-67 (Spearmans's $\rho=0.271, P=0.190$ ) (Fig. 3). We also analyzed the Krenning and NETPET score of the lesions (Table 2). There was a statistically significant correlation between both Krenning score and Ki-67 $(P=0.013)$ and NETPET score and Ki-67 $(P=0.036)(n=43)$.

\section{Correlation between ${ }^{18} \mathrm{~F}$-FDG-PET/CT and grade}

${ }^{18} \mathrm{~F}$-FDG-PET/CT was positive for 11 of 31 patients (35\%) and the $\mathrm{SUV}_{\max }$ ranged from 3.0 to 18.6 (median 4.5, IQR 3.4-8.7). Clinical characteristics of patients according to ${ }^{18}$ F-FDG-uptake are summarized in Table 3 and lesion characteristics are detailed in Fig. 2. In the lesion based analysis on ${ }^{18} \mathrm{~F}$-FDG-PET/CT, 52 primary lesions were analyzed on 31 patients (Fig. 2). Three of the ${ }^{18} \mathrm{~F}$-FDG-avid tumors were G1 (two T3N0 $\varnothing \geq 9 \mathrm{~cm}$ and one T3N1), five G2 (one T1N0, one T2N0, one T3N0, one T3N1 and one patient assessed by EUS-FNB, too frail for surgery) and one well-differentiated G3 (T3N0) tumor (Fig. 4). Two ${ }^{18} \mathrm{~F}$ FDG-positive tumors are intensively followed up without a histological confirmation. ${ }^{18} \mathrm{~F}$-FDG-PET/CT was positive in $19 \%(3 / 16)$ of G1 tumors, $63 \%(5 / 8)$ of G2 tumors and $100 \%(1 / 1)$ of G3 tumors. There was a statistically significant positive correlation between ${ }^{18} \mathrm{~F}$-FDG-uptake and the tumor Ki-67 (Spearmans's $\rho=0.458, P=0.021$ ) (Fig. 3). When tumors were divided according to ${ }^{18} \mathrm{~F}$-FDGpositivity and ${ }^{18} \mathrm{~F}$-FDG-negativity, the correlation with the grade stayed significant $(P=0.023)$ (Table 3$).{ }^{18} \mathrm{~F}$ FDG-positive tumors were significantly larger than ${ }^{18} \mathrm{~F}$-FDG-negative tumors, with a mean maximum diameter of $46 \mathrm{~mm}$ (median 33, IQR 23-90 mm) compared with $24 \mathrm{~mm}$ (median 20, IQR $12-30 \mathrm{~mm} ; P=0.012$ ). All the ${ }^{18} \mathrm{~F}$-FDG-negative patients were asymptomatic and 




Fig. 3 Logarithm variation of the relationship between the tumor Ki-67 and uptake of ${ }^{68}$ Ga-DOTANOC (black circles) and ${ }^{18}$ F-FDG (grey circles) for every tumor

$73 \%{ }^{18}$ F-FDG-positive patients were asymptomatic $(P=$ 0.037). The symptomatic patients had more intense ${ }^{18} \mathrm{~F}$ FDG-uptake than the asymptomatic patients $(P=0.003)$. However, there was no significant correlation between symptoms and grade $(P=0.595)$ or symptoms and Ki-67 ( $P$ $=0.102) .{ }^{18}$ F-FDG-PET/CT showed a positive predictive value (PPV) of $78 \%$ in the detection of potentially aggressive tumors (G2, G3, LN + or M+ histologically confirmed PNETs) and the negative predictive value (NPV) was $69 \%$.

\section{Local and distant metastases}

Six patients had histopathologically confirmed lymph node metastases (mean $4.8 \pm 3.4$ nodes). Three $\mathrm{LN}+$ were in patients with G1 NF-PNETs and three in patients with

Table 2 Krenning and NETPET score of the lesions $(n=43)$

\begin{tabular}{cl}
\hline Krenning score & $n(\%)$ \\
\hline 1 & $1(2)$ \\
2 & $4(9)$ \\
3 & $24(56)$ \\
4 & $14(33)$ \\
NETPET score & $n(\%)$ \\
P1 & $32(75)$ \\
P2 & $8(19)$ \\
P3 & $1(2)$ \\
P4 & $1(2)$ \\
P5 & $1(2)$ \\
\hline
\end{tabular}

From 53 lesions, 43 were analyzed. Two microadenomas, $1{ }^{68} \mathrm{Ga}$-DOTANOCpositive lymph node, and $7{ }^{68} \mathrm{Ga}$-DOTANOC-negative false negative lesions of a patient with 13 NETs were not included to this analysis
G2 NF-PNETs. All the LN+ patients were asymptomatic and sporadic. Two of these six patients with $\mathrm{LN}+(33 \%)$ had an ${ }^{18} \mathrm{~F}$-FDG-positive tumor and four tumors with $\mathrm{LN}+$ were ${ }^{18} \mathrm{~F}$-FDG-negative (67\%). Thus, $18 \%(2 / 11)$ of ${ }^{18}$ F-FDG-patients had LN+ vs. $20 \%(4 / 20)$ of ${ }^{18}$ F-FDGnegative patients had $\mathrm{LN}+$ (Fig. 5). In patients with $\mathrm{LN}+$, the mean primary tumor size was $54 \pm 25 \mathrm{~mm}$ (range 24$90 \mathrm{~mm}$ ). In five patients, preoperative ${ }^{68} \mathrm{Ga}$-DOTANOCPET/CT did not reveal LN metastases. One patient had several LN metastases but ${ }^{68}$ Ga-DOTANOC-PET/CT showed only one metastatic lesion. Two patients were diagnosed with a NF-PNET in the tail of the pancreas and liver metastasis (T3N1M1/G2/Ki-67 10\% and T2N1M1/ G2/Ki-67 3\%). Liver resection is arranged for another patient but to another no liver resection was performed due to progression of the disease.

\section{Prognosis and PET outcome}

During the mean follow-up of $31.3 \pm 6.2$ months, one patient died (due to long-term surgical complications). He had a ${ }^{68}$ Ga-DOTANOC-negative and ${ }^{18}$ F-FDG-positive G1 T3N1 tumor. No recurrence had been detected during the follow-up time. One patient with ${ }^{68} \mathrm{Ga}$-DOTANOC-positive and ${ }^{18} \mathrm{~F}$-FDG-negative primary tumor and liver metastases underwent a laparoscopic distal pancreatectomy and splenectomy. Histopathological analysis confirmed a G2 T3N1M1 tumor. Due to the progression of the disease no liver resection was arranged and capecitabine and temozolomide combination therapy and previously Lutetium-177octreotate therapy was performed. One MEN1 patient with ${ }^{68}$ Ga-DOTANOC-positive and ${ }^{18}$ F-FDG-negative primary 
Table 3 Clinical, histopathological and metabolic features of NF-PNETs

\begin{tabular}{|c|c|c|c|c|c|c|}
\hline & $\begin{array}{l}{ }^{18} \mathrm{~F}-\mathrm{FDG} \text {-positive } \\
\mathrm{SUV}_{\max } \text { range 3.0-8.6 }\end{array}$ & $\begin{array}{l}{ }^{18} \mathrm{~F}-\mathrm{FDG} \text {-negative } \\
\mathrm{SUV}_{\max } \text { range } 0.8-3.2\end{array}$ & $P$ & $\begin{array}{l}{ }^{68} \text { Ga-DOTANOC-positive } \\
\text { SUV }_{\max } \text { range } 8.7-104.7\end{array}$ & $\begin{array}{l}{ }^{68} \mathrm{Ga}-D O T A N O C \text {-negative } \\
\mathrm{SUV}_{\max } 4.4\end{array}$ & $P$ \\
\hline Sex & & & 1.000 & & & 1.000 \\
\hline Male & 7/11 (64) & $13 / 20(65)$ & & $19 / 30$ & $1 / 1$ & \\
\hline Female & 4/11 (36) & $7 / 20(35)$ & & $11 / 30$ & $0 / 1$ & \\
\hline Age, years & $67(38-78)$ & $68(50-71)$ & 1.000 & $66(47-72)$ & 70 & 0.903 \\
\hline Primary tumor size, mm & $33(23-90)$ & $20(13-30)$ & 0.023 & $24(14-38)$ & 33 & 0.581 \\
\hline Asymptomatic & 8/11 (73) & $20 / 20(100)$ & 0.037 & $27 / 30$ & $1 / 1$ & 1.000 \\
\hline $\mathrm{CgA}$ & & & 0.258 & & & 0.516 \\
\hline Strongly positive & 2/11 (18) & $1 / 20(5)$ & & $3 / 30$ & $0 / 1$ & \\
\hline Weakly positive & $6 / 11(55)$ & $9 / 20(45)$ & & $15 / 30$ & $0 / 1$ & \\
\hline Negative & $3 / 11(27)$ & $11 / 20(50)$ & & $12 / 30$ & $1 / 1$ & \\
\hline Location & & & 0.630 & & & 1.000 \\
\hline Head & $6 / 11(55)$ & $6 / 20(30)$ & & $11 / 30$ & $1 / 1$ & \\
\hline Body & 0/11 (0) & $2 / 20(10)$ & & $2 / 30$ & $0 / 1$ & \\
\hline Tail & $3 / 11(27)$ & $7 / 20(35)$ & & $10 / 30$ & $0 / 1$ & \\
\hline Multifocal & 2/11 (18) & $5 / 20(25)$ & & $7 / 30$ & $0 / 1$ & \\
\hline Grade* & & & 0.023 & & & 1.000 \\
\hline G1 & 3/9 (33) & $13 / 16(81)$ & & $15 / 24$ & $1 / 1$ & \\
\hline $\mathrm{G} 2$ & $5 / 9(56)$ & 3/16 (19) & & $8 / 24$ & $0 / 1$ & \\
\hline G3 & $1 / 9(11)$ & $0 / 16(0)$ & & $1 / 24$ & $0 / 1$ & \\
\hline Ki-67 & $4.0(1.5-11.0)$ & $2.0(1.0-2.5)$ & 0.051 & $2.0(1.0-4.5)$ & 1.0 & 0.384 \\
\hline${ }^{68} \mathrm{Ga}$-DOTANOC-positive & 10/11 (91\%) & $20 / 20(100 \%)$ & 0.355 & & & \\
\hline${ }^{18}$ FDG-positive & & & & 10/30 (33\%) & $1 / 1(100 \%)$ & 0.355 \\
\hline $\mathrm{LN}+{ }^{\%}$ & 2/8 (33) & 4/11 (36) & 1.000 & $5 / 30$ & $1 / 1$ & 0.316 \\
\hline
\end{tabular}

Categorical variables are expressed as $n(\%)$ and continuous variables are reported as median values (IQR)

Abbreviations: CgA, circulating chromogranin A, Strongly positive indicates S-CgA $=13.5 \mathrm{nmol} / \mathrm{L}$ or P-CgA 9-37 nmol/L, weakly positive indicates S-CgA $2.2-4.7$

$\mathrm{nmol} / \mathrm{L}$ or $\mathrm{P}-\mathrm{CgA} 3.0-4.8 \mathrm{nmol} / \mathrm{L}$ and negative indicates $\mathrm{S}-\mathrm{CgA}<2.1 \mathrm{nmol} / \mathrm{L}$ or $\mathrm{P}-\mathrm{CgA}<3.0 \mathrm{nmol} / \mathrm{L} ; \mathrm{LN}+$, lymph node metastases

*Lesion analysis (histologically confirmed, $n=25$ )

${ }^{\%}$ Follow-up patients, biopsied patients and one patient who underwent enucleation were excluded because it was not possible to assess their LN+

tumor underwent distal pancreatectomy and splenectomy and histological analysis revealed a G2 T2N1M1 tumor. Currently, a liver resection is planned due to the residual liver metastasis. One patient underwent robotic distal pancreatectomy and splenectomy due to two sporadic T1N0 G1 tumors. Stable residual tumor at the body of the pancreas is followed up. Among the follow-up group the VHL patient's tumor has enlarged and uptake of ${ }^{18} \mathrm{~F}$-FDG increased and surgery is considered. No disease progression has been detected in other patients belonging to the followup group (9 patients, 11 lesions).

\section{Discussion}

To our knowledge, this is the first prospective study evaluating the impact of combined ${ }^{68} \mathrm{Ga}$-DOTANOC and ${ }^{18} \mathrm{~F}$ FDG-PET/CT in predicting the aggressive behavior of NFPNET. There is some previous data of the dual-tracer imaging with series of different gastroenteropancreatic neuroendocrine tumors $[18,24]$ and retrospective series of
PNETs [17, 25]. The main interest of this study was to evaluate if the malignant potential of NF-PNETs could be predicted with dual-tracer PET/CT imaging. We found that there was a statistically significant positive correlation between ${ }^{18} \mathrm{~F}$-FDG-uptake and the proliferation index of the tumor $(P=0.021)$. However, there was no negative correlation between ${ }^{68} \mathrm{Ga}$-DOTANOC-uptake and the Ki-67index $(P=0.190)$. Both the Krenning score and NETPET score correlated statistically significantly with the Ki-67. In this patient cohort, the lower ${ }^{68} \mathrm{Ga}$-DOTANOC uptake, expressed as $\mathrm{SUV}_{\max }$ values, was not directly associated to more aggressive phenotype unless the comparison was made to physiological organ activity by using Krenning score or combined to ${ }^{18} \mathrm{~F}$-FDG-imaging in NETPET score. We also studied the ability of the dual-tracer PET/CT to assess $\mathrm{LN}+$ but no effect was seen in order to enable a diagnosis of a local metastatic disease.

${ }^{18}$ F-FDG-PET/CT imaging has traditionally been used to assess adenocarcinoma and poorly-differentiated G3 

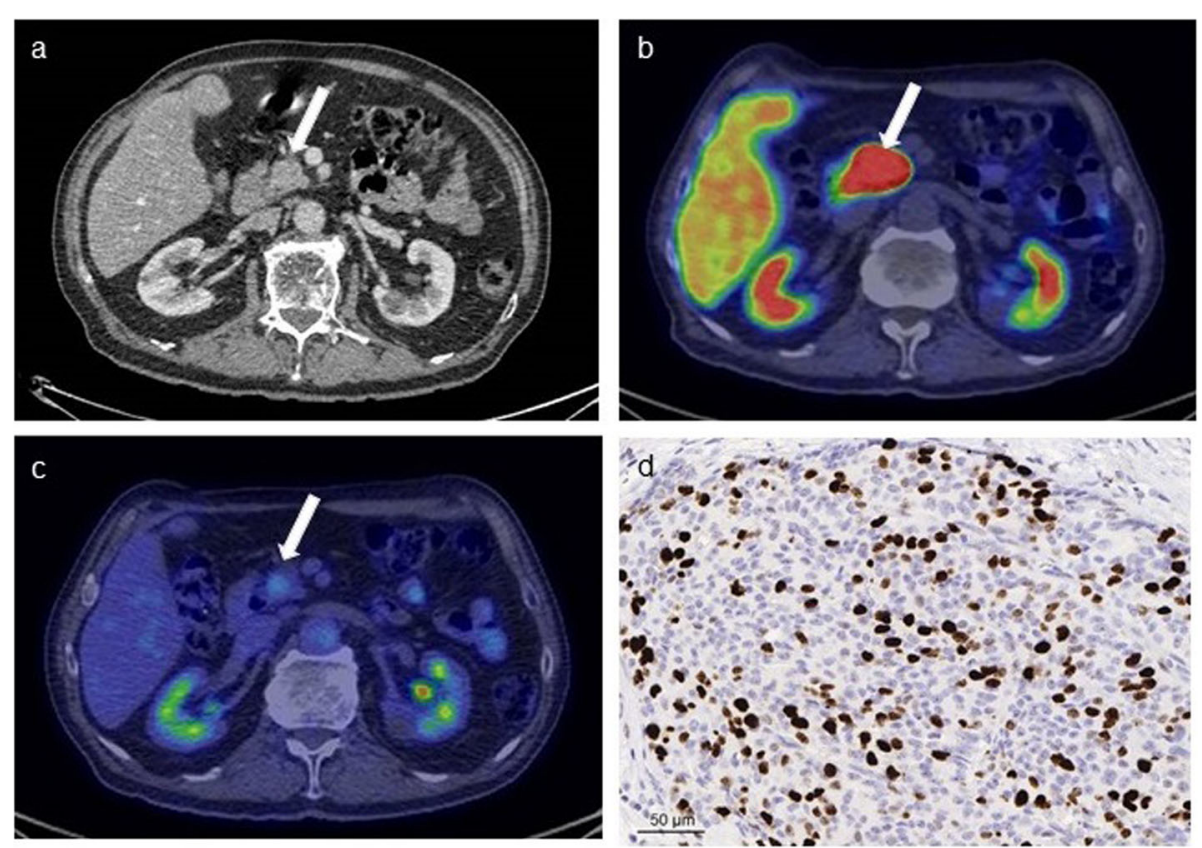

Fig. 4 An 80-year-old male patient had a $3 \mathrm{~cm}$ solid mass in the head of the atrophic pancreas on CT (a). ${ }^{68} \mathrm{Ga}-\mathrm{DOT} A \mathrm{ANOC}-\mathrm{PET} / \mathrm{CT}$ (b) showed intense uptake (SUV $\max 79 \mathrm{~g} / \mathrm{ml})$ and ${ }^{18} \mathrm{~F}-\mathrm{FDG}-\mathrm{PET} / \mathrm{CT}$ (c) was positive (SUV $\max 3.4 \mathrm{~g} / \mathrm{ml}$, higher than liver background). He underwent a pancreaticoduodenectomy and histopathological analysis revealed a well-differentiated G3 PNET (Ki-67 $31 \%)(\mathbf{d}$, image magnification $\times$ 40) without lymph node metastases
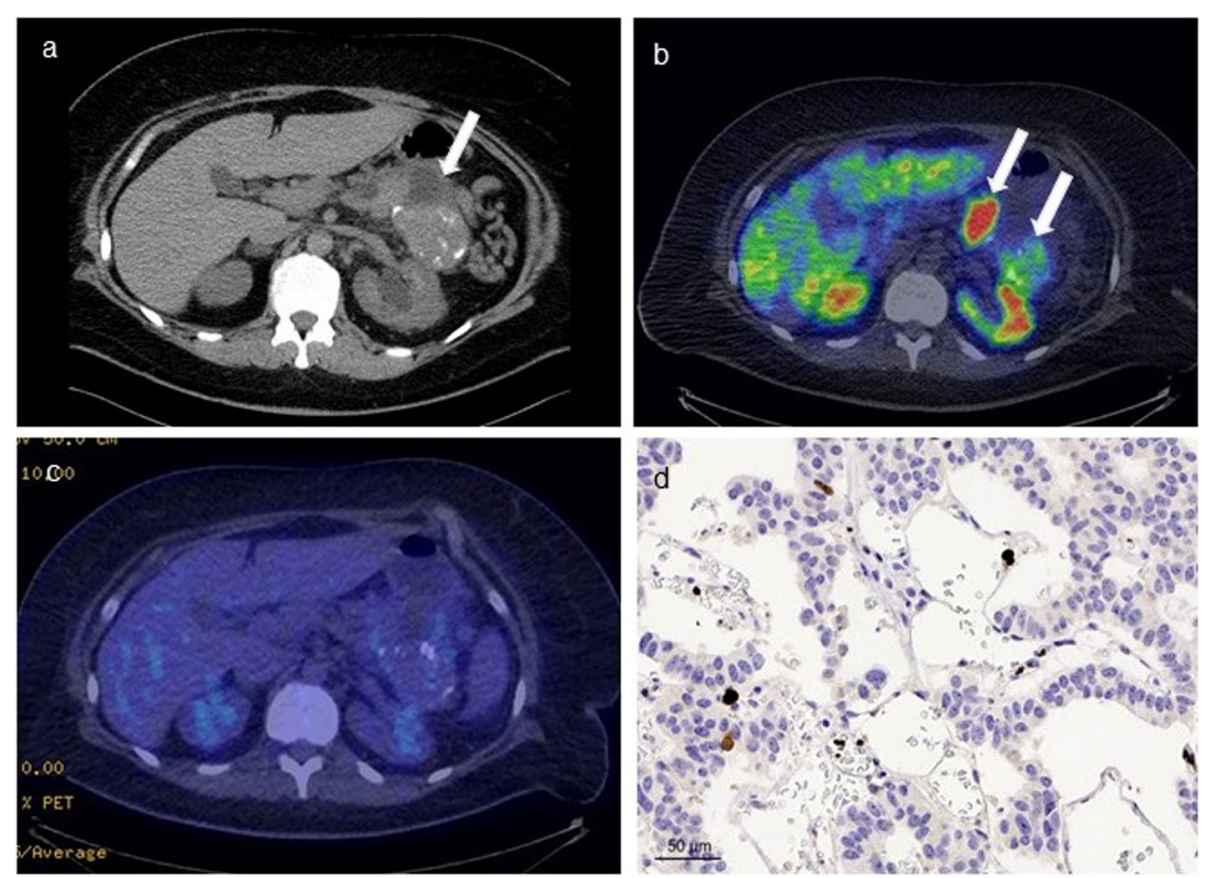

Fig. 5 A 32-year-old female patient had a $10 \mathrm{~cm}$ complex cystic-solid mass in the tail of the pancreas on CT (a). ${ }^{68} \mathrm{Ga}$-DOTANOC-PET/CT (b) showed two different uptake intensity areas around a large cystic component (SUV $\max 29 \mathrm{~g} / \mathrm{ml}$ and $10.6 \mathrm{~g} / \mathrm{ml}$ ) in the pancreatic tumor. ${ }^{18} \mathrm{~F}$-FDGPET/CT (c) was negative $\left(S_{U} V_{\max } 3.2 \mathrm{~g} / \mathrm{ml}\right.$ ). She underwent a distal pancreatectomy and splenectomy and histopathological analysis revealed a G1 PNET (Ki-67 < 2\%) (d, image magnification $\times 40)$ with five lymph node metastases 
neuroendocrine carcinomas (NECs). However, recent data has shown that ${ }^{18} \mathrm{~F}$-FDG-PET/CT has prognostic value in patients with well-differentiated NET, showing that high uptake is associated with increased risk of early progression $[5,26]$. In retrospective series the prognostic value of ${ }^{18} \mathrm{~F}$ FDG-PET/CT imaging was even more powerful than the grade of the tumor $[15,16]$. A study by Chan et al. [23] of 62 metastatic NET patients combined the results of somatostatin analogue and ${ }^{18}$ F-FDG-PET/CT to obtain an imaging biomarker, a NETPET-score (grades P1-5), and a classification system reflecting the burden of the disease. Grade P1 indicated purely somatostatin analogue avid lesion without ${ }^{18} \mathrm{~F}$-FDG-uptake and P5 indicated the presence of a significant ${ }^{18} \mathrm{~F}$-FDG-positive and somatostatin analogue-negative disease. The NETPET-score correlated positively with the histopathological grade and offered a better prediction of overall survival. In concordance with this we found a positive correlation between the NETPET score and the proliferation index. Further there was a correlation between the $\mathrm{SUV}_{\max }$ of ${ }^{18} \mathrm{~F}$-FDG-PET/CT and the proliferation index, and this correlation maintained significance when the patients were divided into $\mathrm{WHO}$ grades by Ki-67. Sharma et al. [27] concluded that $\mathrm{SUV}_{\max }$ on ${ }^{68} \mathrm{Ga}$ DOTANOC-PET/CT was an independent, positive prognostic factor in patients with well-differentiated NET in a two-year median follow-up time. However, in light of this observation, we did not find significant prognostic role for ${ }^{68} \mathrm{Ga}$-DOTANOC-uptake in our study due to short followup time.

SSTR-based functional imaging with ${ }^{68} \mathrm{Ga}$-labelled peptides, ${ }^{68} \mathrm{Ga}$-DOTATATE, ${ }^{68} \mathrm{Ga}$-DOTATOC, and ${ }^{68} \mathrm{Ga}$ DOTANOC, has recently become the gold standard in the diagnosis of the NETs due to better sensitivity compared to ${ }^{111}$ In-DTPA-octreotide (Octreoscan ${ }^{\circ}$ ) [28] and lower patient radiation and higher spatial resolution than SPECT/CT [29]. ${ }^{68} \mathrm{Ga}$-DOTATATE has the strongest SSTR2-binding affinity. However, ${ }^{68}$ Ga-DOTATOC and especially ${ }^{68} \mathrm{Ga}$-DOTANOC have wider affinity profiles, including SSTR2, SSTR3 and SSTR5. The three analogs have shown no differences in clinical practice.

The sensitivity of the ${ }^{68} \mathrm{Ga}$-DOTANOC PET/CT for detecting NF-PNETs is high and SSTR-based imaging is a mandatory procedure to guide treatment planning [30]. In our series, the sensitivity was $97 \%$ and the smallest ${ }^{68} \mathrm{Ga}$-DOTANOC-avid and histologically confirmed tumor was $6 \mathrm{~mm}$. Lesion-based analysis sensitivity was 85\% (45/53). Seven FN lesions occurred in the same MEN1 patient who had a total of 13 PNETs (smallest 5$10 \mathrm{~mm}$ in size) in the histopathological analysis. The fact that not all of these multiple lesions of this MEN1 patient were detected with the ${ }^{68} \mathrm{Ga}$-DOTANOC should not be considered a diagnostic failure.

The prognostic value of ${ }^{18} \mathrm{~F}$-FDG-PET/CT in functional imaging of NETs has been a target for recent research. Garin et al. [24] investigated prospectively well-differentiated metastatic NETs and reported that ${ }^{18} \mathrm{~F}$-FDG-positivity correlated with decreased progression free survival and overall survival. Cingarlini et al. [17] compared retrospectively G1 and G2 PNETs and concluded that ${ }^{18} \mathrm{~F}$-FDG-PET/CT was positive in a smaller proportion of G1 tumors (20\%) compared with G2 tumors (76\%). In our study 34\% NF-PNET patients had a positive ${ }^{18} \mathrm{~F}$-FDG-PET/CT and the ${ }^{18} \mathrm{~F}$-FDG-PET/CT showed a PPV of $78 \%$ in the detection of potentially aggressive tumors (G2, G3, LN+ or M+ PNETs), and the NPV was $69 \% .{ }^{18} \mathrm{~F}$-FDG-PET/CT should have some relevance when treatment options are discussed but the possibility of false imaging findings, especially false negative, should be kept in mind. In this prospective study there was a statistically significant correlation between the $\mathrm{SUV}_{\max }$ of ${ }^{18}$ F-FDG-PET/CT and the Ki-67 of the primary tumor.

In the diagnostic workup of NF-PNETs, an endoscopic ultrasonography-guided fine-needle aspiration (EUS-FNA) could be an option, but it does not necessarily represent the whole tumor in terms of aggressiveness and the tissue material may be insufficient. In the French study [31], 30\% of tumor grading was up-scaled on the resected tissue. Additionally, the heterogeneity in the tumor tissue may interfere the assessment of Ki-67-labeling [32]. However, in the prospective, multicenter, randomized controlled trial the EUS-FNB, which also was used in our study, produced a more accurate diagnosis than EUS-FNA [33].

Boninsegna et al. [34] found that $\mathrm{Ki}-67>5 \%$ and the ratio between the number of metastatic LNs above that of the examined LNs were better predictors of the recurrence than tumor size. Further, in our series there was no correlation between the tumor size and Ki-67-index, but the ${ }^{18} \mathrm{~F}$-FDG-positive tumors were significantly larger than the ${ }^{18} \mathrm{~F}$-FDG-negative tumors.

Our study results show that dual-tracer PET/CT imaging has a complementary role and it enables the detection of a potentially aggressive disease during the diagnostic workup on well-differentiated NF-PNETs. Due to this we recommend ${ }^{18} \mathrm{~F}$-FDG-PET/CT to be a part of a systematic diagnostic work-up of asymptomatic NF-PNETs. Especially if a consensus regarding surgical therapy cannot be reached, dual-tracer PET/CT could supplement the evaluation of the nature of the tumor. Due to our results ${ }^{18} \mathrm{~F}$ FDG-avid tumors should be considered for surgery if there are no contraindications. In a case of a ${ }^{18} \mathrm{~F}$-FDG-negative lesion follow-up could be considered if all the other aspects of the diagnostic work-up also support this treatment strategy.

The strengths of the present study were histopathological re-evaluation of all the tumors as well as the immunohistochemical Ki-67 staining and automated image analysis. The major limitation of the present study is the 
small number of patients, as the disease is rare. In addition, the lack of histopathological confirmation of the follow-up patients limits the analysis. For this subgroup of patients, the collection of samples was not technically or ethically feasible. However, the correlation analyses were performed with histopathologically confirmed cases. Since pancreatic neuroendocrine carcinomas are rare, the study population consists of well-differentiated PNETs, which impairs the sensitivity and specificity of ${ }^{18} \mathrm{~F}$-FDG-PET/CT to detect potentially aggressive tumors. In two patients the interval between PET imaging was delayed (244 and 360 d), however, due to the indolent nature of NF-PNETs the long dual PET/CT interval is likely to be of no great importance and we preferred not to exclude these patients. Another limitation is the relatively short follow-up time (mean $31.3 \mathrm{mo}$, SD $6.2 \mathrm{mo}$ ) which is insufficient to estimate a definitive prognosis. There is a possibility that during a longer follow-up time the ${ }^{18} \mathrm{~F}$-FDG-positive tumors in this study would prove to be progressive, and our intent is to analyze long time prognosis. In addition, it would be essential to detect which biological and immunohistochemical features are common for these tumors.

\section{Conclusion}

$\mathrm{SUV}_{\max }$ measured on ${ }^{18} \mathrm{~F}$-FDG-PET/CT was significantly associated with the proliferation index, $\mathrm{Ki}-67$, and will serve as a surrogate measure of tumor aggressiveness in patients with asymptomatic NF-PNET. Further, the ${ }^{68} \mathrm{Ga}$-DOTANOC-PET/CT has notable prognostic value since the Krenning score predicts the histopathological tumor grade.

To conclude, we recommend ${ }^{18}$ F-FDG-PET/CT to be a part of a systematic diagnostic work-up of asymptomatic NF-PNETs. This prospective study shows that dual-tracer PET/CT imaging, using ${ }^{18} \mathrm{Ga}$-DOTANOC and ${ }^{18} \mathrm{~F}$-FDG, has a complementary role and it enables the detection of a potentially aggressive disease during the diagnostic workup on well-differentiated NF-PNETs.

\footnotetext{
Abbreviations

${ }^{18}$ F-FDG: ${ }^{18}$ F-fluoro-2-deoxyglucose; 5-HIAA: 5-hydroxyindoleatic acid; ${ }^{68} \mathrm{Ga}-$ DOTANOC: ${ }^{68} \mathrm{Ga}$-DOTA-1-Nal ${ }^{3}$-octreotide; ${ }^{68} \mathrm{Ga}$-DOTATATE: ${ }^{68} \mathrm{Ga}$-DOTA-Tyr ${ }^{3}$ octreotate; ${ }^{68} \mathrm{Ga}$-DOTATOC: ${ }^{68} \mathrm{Ga}$-DOTA-Tyr ${ }^{3}$-octreotide; ADW: Advantage Workstation for Diagnostic Imaging; BMl: Body mass index; Bq: Becquerel; CgA: Chromogranin A; CT: Computed tomography; EUS-FNA: Endoscopic ultrasonography-guided fine-needle aspiration; EUS-FNB: Endoscopic ultrasonography and fine-needle biopsy; FN: False negative; G: Grade; IQR: Interquartile range; LN: Lymph node; LN+: Lymph node metastases; M+: Distant metastases; MEN: Multiple endocrine neoplasia; MIB: Monoclonal antibody against the Ki-67 antigen; MRI: Magnetic resonance imaging; NEC: Poorly-differentiated grade 3 neuroendocrine carcinoma; NF-PNET: Nonfunctional pancreatic neuroendocrine tumor; NPV: Negative predictive value; Octreoscan ${ }^{\oplus}:{ }^{111}$ In-DTPA-octreotide; PET/CT: Positron emission tomography/ computed tomography; PP: Pancreatic polypeptide; PPV: Positive predictive value; SD : Standard deviation; SSTR: Somatostatin receptor; SUV $V_{\text {max: }}$ Maximum standardized uptake value; VHL: Von Hippel-Lindau; WHO: World Health Organization
}

\section{Acknowledgements}

We would like to thank the staff of the Turku and Helsinki PET Centres. We would also like to thank Pälvi Vento, Tora Bäckström and Antti Jekunen for their contribution to this work. We would also like to thank The Finnish Cancer Foundation, The Sigrid Juselius Foundation, The Mary and Georg Ehrnrooth Foundation.

\section{Authors' contributions}

SM operated as a principal investigator, participated in patient recruitment, data collection and analysis and drafted the manuscript. HS has a major role in design and conceiving the study, patient recruitment, data collection and revising of the manuscript. JK had a major role in conception and design of the study (especially PET/CT protocol) and helped to draft the manuscript. JaS participated in conceiving the study and blindly evaluated the histopathological specimens and helped revising the manuscript. C S-J participated in the design of the study and to the patient recruitment and helped to revise the manuscript. RG participated in conceiving the design of the study and patient recruitment and helped to revise the manuscript. JuS participated in the design of the study (especially PET/CT protocol) and transformation of the imaging data between the hospitals. HM performed the statistical analysis. TV carried out the histopathological analyses (Ki-67 analyses with Immunoratio software) and participated in drafting and revising of the manuscript. JA participated in design of the study (especially pathological point of view) and coordinated histopathological analyses and blindly analyzed the pathological specimens and helped to revise the manuscript. SK operated as a second principal investigator, conceived of the study, and participated its design and coordination and patient recruitment and helped to draft and revise the manuscript. All authors read and approved the final manuscript.

\section{Funding}

This was funded by the Research Funding of Turku University Central Hospital. S.M has received grants from the Education and Research foundation of Turku University Hospital (TYKS saatio); and the Finnish Medical Foundation (Suomen Laaketieteen Saatio). C. S-J has received a grant from Finska Lakaresallskapet.

\section{Availability of data and materials}

The datasets used and/or analyzed during the current study are available from the corresponding author on reasonable request.

\section{Ethics approval and consent to participate}

The study was conducted according to the Guidelines of the Declaration of Helsinki, and the study protocol was approved by the Ethics Committee of the Hospital District of Southwest Finland and by Turku Clinical Research Centre. All the patients gave their written informed consent before participating in the study.

\section{Consent for publication}

Not applicable

\section{Competing interests}

The authors declare that they have no competing interests.

\section{Author details}

'Division of Digestive Surgery and Urology, Turku University Hospital, P.O. Box 52, FIN-20521 Turku, Finland. ${ }^{2}$ Turku PET Centre, Turku University Hospital, P.O. Box 52, FIN-20521 Turku, Finland. ${ }^{3}$ Department of Abdominal Surgery, Translational Cancer Medicine Research Program, Faculty of Medicine, University of Helsinki and Helsinki University Hospital, P.O. Box 340, FIN-00029 Helsinki, Finland. ${ }^{4}$ Department of Clinical Physiology and Nuclear Medicine, Turku University Hospital, P.O. Box 52, FIN-20521 Turku, Finland.

${ }^{5}$ Department of Pathology, Turku University Hospital, P.O. Box 52, FIN-20521 Turku, Finland. ${ }^{6}$ Department of Endocrinology, Abdominal Center, University of Helsinki and Helsinki University Hospital, P.O. Box 340, FIN-00029 Helsinki, Finland. ${ }^{7}$ Department of Clinical Physiology and Nuclear Medicine, Helsinki University Hospital, Haartmaninkatu 4, FIN-00029 Helsinki, Finland.

${ }^{8}$ Department of Surgery, University of Helsinki and Helsinki University Hospital, P.O. Box 340, FIN-00029 Helsinki, Finland. ${ }^{9}$ HUSLAB, Department of Pathology, University of Helsinki and Helsinki University Hospital, P.O. Box 
400, FIN-00029 Helsinki, Finland. ${ }^{10}$ Institute for Molecular Medicine Finland (FIMM), HiLIFE, University of Helsinki, P.O. Box 20, Fl-00014 Helsinki, Finland.

\section{Received: 6 October 2019 Accepted: 10 December 2019} Published online: 23 December 2019

\section{References}

1. Yao JC, Hassan M, Phan A, Dagohoy C, Leary C, Mares JE, Abdalla EK, Fleming JB, Vauthey J, Rashid A, Evans DB. One hundred years after "carcinoid": epidemiology of and prognostic factors for neuroendocrine tumors in 35,825 Cases in the United States. JCO. 2008;26:3063-72. https:// doi.org/10.1200/JCO.2007.15.4377.

2. Cheema A, Weber J, Strosberg J. Incidental detection of pancreatic neuroendocrine tumors: an analysis of incidence and outcomes. Ann Surg Oncol. 2012;19:2932-6. https://doi.org/10.1245/s10434-012-2285-7.

3. Fischer L, Kleeff J, Esposito I, Hinz U, Zimmermann A, Friess H, Buchler MW Clinical outcome and long-term survival in 118 consecutive patients with neuroendocrine tumours of the pancreas. Br J Surg. 2008;95:627-35. https:// doi.org/10.1002/bjs.6051

4. Bilimoria KY, Talamonti MS, Tomlinson JS, Stewart AK, Winchester DP, Ko CY, Bentrem DJ. Prognostic score predicting survival after resection of pancreatic neuroendocrine tumors: analysis of 3851 patients. Ann Surg. 2008;247:490-500. https://doi.org/10.1097/SLA.0b013e31815b9cae.

5. Falconi M, Eriksson B, Kaltsas G, Bartsch DK, Capdevila J, Caplin M, Kos-Kudla B, Kwekkeboom D, Rindi G, Kloppel G, Reed N, Kianmanesh R, Jensen RT. Consensus guidelines update for the management of functional p-NETs ( $F$ p-NETs) and non-functional p-NETs (NF-p-NETs). Neuroendocrinology. 2016; 103:153-71. https://doi.org/10.1159/000443171.

6. Partelli S, Cirocchi R, Crippa S, Cardinali L, Fendrich V, Bartsch DK, Falconi M. Systematic review of active surveillance versus surgical management of asymptomatic small non-functioning pancreatic neuroendocrine neoplasms. Br J Surg. 2017;104:34-41. https://doi.org/10.1002/bjs.10312

7. Kim MJ, Choi DW, Choi SH, Heo JS, Park HJ, Choi KK, Jang K, Sung J. Surgical strategies for non-functioning pancreatic neuroendocrine tumours. $\mathrm{Br} J$ Surg. 2012;99:1562-8. https://doi.org/10.1002/bjs.8892.

8. Hashim YM, Trinkaus KM, Linehan DC, Strasberg SS, Fields RC, Cao D, Hawkins WG. Regional lymphadenectomy is indicated in the surgical treatment of pancreatic neuroendocrine tumors (pnets). Ann Surg. 2014;259: 197-203. https://doi.org/10.1097/SLA.0000000000000348.

9. Partelli S, Boninsegna L, Crippa S, Falconi M, Gaujoux S, Cherif R, Scarpa A, Sauvanet A, Couvelard A, Ruszniewski P. Pattern and clinical predictors of lymph node involvement in nonfunctioning pancreatic neuroendocrine tumors (NF-PanNETs). JAMA Surgery. 2013;148:932-9.

10. Schafer M, Mullhaupt B, Clavien P. Evidence-based pancreatic head resection for pancreatic cancer and chronic pancreatitis. Ann Surg. 2002:236:137

11. Sauvanet A, Partensky C, Sastre B, Gigot J, Fagnies P, Tuech J, Letoublon C. Medial pancreatectomy: a multi-institutional retrospective study of 53 patients by the French Pancreas Club. Surgery. 2002;132:836-43.

12. Virgolini I, Ambrosini V, Bomanji JB, Baum RP, Fanti S, Gabriel M, Papathanasiou ND, Pepe G, Oyen W, Cristoforo CD, Chiti A. Procedure guidelines for PET/CT tumour imaging with 68Ga-DOTA-conjugated peptides: 68Ga-DOTA-TOC, 68Ga-DOTA-NOC, 68Ga-DOTA-TATE. Eur J Nucl Med Mol Imaging. 2010;37:2004-10. https://doi.org/10.1007/s00259010-1512-3.

13. Sharma P, Arora S, Dhull VS, Naswa N, Kumar R, Ammini AC, Bal C. Evaluation of $68 \mathrm{Ga}-\mathrm{DOTANOC}$ PET/CT imaging in a large exclusive population of pancreatic neuroendocrine tumors. Abdom Imaging. 2015;40: 299-309. https://doi.org/10.1007/s00261-014-0219-5.

14. Naswa N, Sharma P, Kumar A, Nazar AH, Kumar R, Chumber S, Bal C. Gallium-68-DOTA-NOC PET/CT of patients with gastroenteropancreatic neuroendocrine tumors: a prospective single-center study. AJR Am J Roentgenol. 2011;197:1221-8. https://doi.org/10.2214/AJR.11.7298.

15. Binderup T, Knigge U, Loft A, Federspiel B, Kjaer A. 18F-fluorodeoxyglucose positron emission tomography predicts survival of patients with neuroendocrine tumors. Clin Cancer Res. 2010;16:978-85. https://doi.org/10. 1158/1078-0432.CCR-09-1759.

16. Johnbeck CB, Knigge U, Langer SW, Loft A, Berthelsen AK, Federspiel B, Binderup T, Kjaer A. Prognostic value of 18F-FLT PET in patients with neuroendocrine neoplasms: a prospective head-to-head comparison with 18F-FDG PET and Ki-67 in 100 patients. Journal of nuclear medicine: official publication, Society of Nuclear Medicine. 2016;57:1851-7. https://doi.org/10. 2967/jnumed.116.174714.

17. Cingarlini S, Ortolani S, Salgarello M, Butturini G, Malpaga A, Malfatti V, D'Onofrio M, Davì MV, Vallerio P, Ruzzenente A, Capelli P, Citton E, Grego E, Trentin C, De Robertis R, Scarpa A, Bassi C, Tortora G. Role of combined 68Ga-DOTATOC and 18F-FDG positron emission tomography/computed tomography in the diagnostic workup of pancreas neuroendocrine tumors. Pancreas. 2017:46:42-7. https://doi.org/10.1097/MPA.0000000000000745.

18. Panagiotidis E, Alshammari A, Michopoulou S, Skoura E, Naik K, Maragkoudakis E, Mohmaduvesh M, Al-Harbi M, Belda M, Caplin ME, Toumpanakis C, Bomanji J. Comparison of the impact of 68 Ga-DOTATATE and 18 F-FDG PET/CT on clinical management in patients with Neuroendocrine tumors. J Nucl Med. 2017:58:91-8.

19. Partelli S, Bartsch DK, Capdevila J, Chen J, Knigge U, Niederle B, Dijkum, Els J. M. Nieveen van, Pape U, Pascher A, Ramage J, Reed N, Ruszniewski P, Scoazec J, Toumpanakis C, Kianmanesh R, Falconi M, Participants, all other Antibes Consensus Conference. ENETS Consensus Guidelines for the Standards of Care in Neuroendocrine Tumours: Surgery for Small Intestinal and Pancreatic Neuroendocrine Tumours. NEN. 2017;105:255-265. doi: https://doi.org/10.1159/000464292.

20. Tuominen $V$, Ruotoistenmaki S, Viitanen A, Jumppanen M, Isola J. ImmunoRatio: a publicly available web application for quantitative image analysis of estrogen receptor (ER), progesterone receptor (PR), and Ki-67. Breast Cancer Res. 2010;12:R56. https://doi.org/10.1186/bcr2615.

21. Remes SM, Tuominen VJ, Helin H, Isola J, Arola J. Grading of neuroendocrine tumors with $\mathrm{Ki}-67$ requires high-quality assessment practices. Am J Surg Pathol. 2012;36:1359-63. https://doi.org/10.1097/PAS. 0b013e3182632038.

22. Krenning EP, Valkema R, Kooij PP, Breeman WA, Bakker WH, de Herder WW, van Eijck CH, Kwekkeboom DJ, de Jong M, Pauwels S. Scintigraphy and radionuclide therapy with [indium- 111-labelled-diethyl triamine pentacetic acid-D-Phe1]-octreotide. Ital J Gastroenterol Hepatol. 1999;31:219-23.

23. Chan DL, Pavlakis N, Schembri GP, Bernard EJ, Hsiao E, Hayes A, Barnes T, Diakos C, Khasraw M, Samra J, Eslick E, Roach PJ, Engel A, Clarke SJ, Bailey DL. Dual Somatostatin Receptor/FDG PET/CT Imaging in Metastatic Neuroendocrine Tumours: Proposal for a Novel Grading Scheme with Prognostic Significance. Theranostics. 2017;7:1149-58.

24. Garin E, Le Jeune F, Devillers A, Cuggia M, de Lajarte-Thirouard A, Bouriel C, Boucher E, Raoul J. Predictive value of 18F-FDG PET and Somatostatin receptor scintigraphy in patients with metastatic endocrine tumors. The Journal of Nuclear Medicine. 2009;50:858-64. https://doi.org/10.2967/ jnumed.108.057505.

25. Mapelli P, Partelli S, Salgarello M, Muffatti F, Rancoita PM, Pasetto S, Gianolli $L$, Falconi M, Picchio M. Risk stratification in pancreatic neuroendocrine tumours: the role of combined 68Ga-DOTATOC and 18F-FDG PET/CT. J Nucl Med. 2018;59:1303.

26. Bahri $H$, Laurence L, Edeline J, Leghzali H, Devillers A, Raoul J, Cuggia M, Mesbah H, Clement B, Boucher E, Garin E. High prognostic value of 18F-FDG PET for metastatic gastroenteropancreatic neuroendocrine tumors: a long-term evaluation. Journal of nuclear medicine : officia publication, Society of Nuclear Medicine. 2014;55:1786-90. https://doi. org/10.2967/jnumed.114.144386.

27. Sharma P, Naswa N, KC S, Alvarado L, Dwivedi A, Yadav $Y$, Kumar R, Ammini A, Bal C. Comparison of the prognostic values of 68Ga-DOTANOC PET/CT and 18F-FDG PET/CT in patients with well-differentiated neuroendocrine tumor. Eur J Nucl Med Mol Imaging. 2014;41:2194-202. https://doi.org/10. 1007/s00259-014-2850-3.

28. Krausz Y, Freedman N, Rubinstein R, Lavie E, Orevi M, Tshori S, Salmon A, Glaser B, Chisin R, Mishani E, Gross DJ. ${ }^{68}$ Ga-DOTA-NOC PET/CT Imaging of Neuroendocrine Tumors: Comparison with ${ }^{111}$ In-DTPA-Octreotide (OctreoScan ${ }^{\circledast}$ ). Mol Imaging Biol. 2011;13:583-93.

29. Gabriel M, Decristoforo C, Kendler D, Dobrozemsky G, Heute D, Uprimny C, Kovács P, Von Guggenberg E, Bale R, Virgolini IJ. 68Ga-DOTA-Tyr3-octreotide PET in neuroendocrine tumors: Comparison with somatostatin receptor scintigraphy and CT. J Nucl Med. 2007:48:508-18.

30. Ambrosini V, Campana D, Bodei L, Nanni C, Castellucci P, Allegri V, Montini GC, Tomassetti P, Paganelli G, Fanti S. 68Ga-DOTANOC PET/CT Clinical Impact in Patients with Neuroendocrine Tumors. J Nucl Med. 2010;51:66973. https://doi.org/10.2967/jnumed.109.071712.

31. Rebours V, Cordova J, Couvelard A, Fabre M, Palazzo L, Pierre Vullierme M, Hentic O, Sauvanet A, Aubert A, Bedossa P, Ruszniewski P. Can pancreatic 
neuroendocrine tumour biopsy accurately determine pathological characteristics? Dig Liver Dis. 2015;47:973-7. https://doi.org/10.1016/j.dld. 2015.06.005.

32. Yang Z, Tang, Klimstra LH, David S. Effect of tumor heterogeneity on the assessment of Ki67 labeling index in well-differentiated neuroendocrine tumors metastatic to the liver: implications for prognostic stratification. Am J Surg Pathol. 2011;35:853-60.

33. Cheng B, Zhang Y, Chen Q, Sun B, Deng Z, Shan H, Dou L, Wang J, Li Y, Yang $X$, Jiang T, Xu G, Wang G. Analysis of fine-needle biopsy vs fine-needle aspiration in diagnosis of pancreatic and Abdominal Masses: A Prospective, Multicenter, Randomized Controlled Trial. Clin Gastroenterol Hepatol. 2018; 16:1314-21. https://doi.org/10.1016/j.cgh.2017.07.010.

34. Boninsegna L, Panzuto F, Partelli S, Capelli P, Fave GD, Bettini R, Pederzoli P, Scarpa A, Falconi M. Malignant pancreatic neuroendocrine tumour: Lymph node ratio and Ki67 are predictors of recurrence after curative resections. Eur J Cancer. 2012;48:1608-15.

\section{Publisher's Note}

Springer Nature remains neutral with regard to jurisdictional claims in published maps and institutional affiliations.

\section{Submit your manuscript to a SpringerOpen ${ }^{\circ}$ journal and benefit from:}

- Convenient online submission

- Rigorous peer review

- Open access: articles freely available online

High visibility within the field

- Retaining the copyright to your article

Submit your next manuscript at $\boldsymbol{\nabla}$ springeropen.com 